\title{
EFEITO DA Gliricidia sepium SOBRE NUTRIENTES DO SOLO, MICROCLIMA E PRODUTIVIDADE DO MILHO EM SISTEMA AGROFLORESTAL NO AGRESTE PARAIBANO(1)
}

\author{
Aldrin Martin Pérez Marin ${ }^{(2)}$, Rômulo Simões César \\ Menezes $^{(3)}$, Emanuel Dias Silva ${ }^{(4)} \&$ Everardo \\ Valadares de Sá Barreto Sampaio(5)
}

\begin{abstract}
RESUMO
Gliricidia sepium é uma leguminosa arbórea que tem sido utilizada em sistemas em aléias no semi-árido nordestino por apresentar bom desenvolvimento em condições de estresse hídrico. Entretanto, há pouca informação disponível sobre o efeito da introdução dessa espécie nos agroecossistemas da região. No presente estudo, objetivou-se avaliar a influência da distância de plantas de Gliricidia sepium sobre características da cultura do milho e do solo e microclima no Agreste Paraibano. O estudo foi realizado no município de Esperança (PB), em área de 0,5 ha, onde, em 1996, foram plantadas fileiras de G. sepium espaçadas 6 m entre si e com $1 \mathrm{~m}$ entre as árvores. Nesta área, em 2002, foram delimitadas quatro parcelas de $6 \times 8 \mathrm{~m}$ e, em cada parcela, foi estabelecido um transeto perpendicular às fileiras de árvores com três posições de amostragem: (1) nas fileiras de árvores (0 m); (2) a $1 \mathrm{~m}$ das fileiras de árvores, e (3) a $3 \mathrm{~m}$ de distância das fileiras de árvores. $\mathrm{O}$ delineamento experimental utilizado foi em blocos casualizados com quatro repetições. A massa seca de folhedo caído embaixo da fileira de árvores foi de $1.390 \mathrm{~kg} \mathrm{ha}^{-}$ ${ }^{1}$ e diminuiu, gradativamente, para $270 \mathrm{~kg} \mathrm{ha}^{-1}$ a $3 \mathrm{~m}$ de distância das árvores. As concentrações de $\mathrm{P}$, K e matéria orgânica leve (MOL) embaixo das árvores foram maiores do que a 1 e $3 \mathrm{~m}$ de distância das fileiras. As médias mensais das temperaturas mínimas do ar e do solo embaixo e a $3 \mathrm{~m}$ das árvores foram similares. Entretanto, as médias mensais das temperaturas máximas do solo e do ar foram de
\end{abstract}

\footnotetext{
(1) Parte do trabalho de Tese de Doutorado do primeiro autor, Universidade Federal de Pernambuco - UFPE. Recebido para publicação em maio de 2005 e aprovado em março de 2006.

${ }^{(2)}$ Doutorando em Tecnologias Energéticas e Nucleares, Grupo de Fertilidade de Solos/Radioagronomia, Departamento de Energia Nuclear (DEN), , Universidade Federal de Pernambuco - UFPE. Av. Prof. Luiz Freire 1000, CEP 50740-540 Recife (PE). Bolsista CNPq. E-mail: perez@ufpe.br

(3) Professor adjunto do Departamento de Energia Nuclear, UFPE. E-mail: rmenezes@ufpe.br

(4) Estudante de Agronomia, Universidade Federal da Paraíba. Bolsista do CNPq. E-mail: emanoeldias@yahoo.com.br

(5) Professor Titular do Departamento de Energia Nuclear, UFPE. Bolsista do CNPq. E-mail: esampaio@ufpe.br
} 
6 e $2{ }^{\circ} \mathrm{C}$ mais altas a $3 \mathrm{~m}$ das árvores, respectivamente, ao longo do período de estudo. A umidade do solo foi significativamente menor embaixo das árvores do que a 1 e $3 \mathrm{~m}$ de distância. O milho produziu mais grãos e palha e acumulou mais nutrientes nas posições mais próximas das fileiras de G. sepium.

Termos de indexação: semi-árido, matéria orgânica leve, umidade do solo.

\title{
SUMMARY: EFFECTS OF GLIRICIDIA SEPIUM ON SOIL NUTRIENTS, MICROCLIMATE AND MAIZE YIELD IN AN AGROFORESTRY SYSTEM IN SEMI-ARID PARAIBA, BRAZIL
}

\begin{abstract}
Gliricidia sepium is a leguminous tree that has been used in alley cropping systems in the Northeastern semi-arid region of Brazil because of its good development under water stress conditions. However, little information is available on the effects of the introduction of this species into regional agroecosystems. The objective of the present study was to evaluate the influence of the distance of Gliricidia sepium plants on the characteristics of maize crop, soil properties and microclimate conditions in the semi-arid region of Paraiba, Brazil. The study was conducted in the Esperança County, Paraíba State, Brazil., where G. sepium trees were planted on a 0.5 ha field in 1996. The spacing used was $6 \mathrm{~m}$ between rows and $1 \mathrm{~m}$ between plants within the rows. In 2002, four $48 \mathrm{~m}^{2}$ plots were established within this field. Within each plot, three sampling positions were established: (1) under tree crowns in the rows (0 m); (2) $1 \mathrm{~m}$ apart from tree rows; and (3) $3 \mathrm{~m}$ apart from the tree rows. The experiment had a randomized block design with four replications. Litterfall dry matter amounted to $1.390 \mathrm{~kg} \mathrm{ha-}$ ${ }^{1}$ under the trees and decreased to $270 \mathrm{~kg} \mathrm{ha}^{-1}$ at a distance of $3 \mathrm{~m}$ from the trees. Soil P, Kand light fraction of organic matter were significantly greater under the trees as compared to those between the tree rows. Soil and air monthly minimum temperature averages were not influenced by the distance from the trees. However, soil and air monthly maximum temperature averages were 6 and $2^{\circ} \mathrm{C}$ lower under the trees, respectively. Soil moisture was significantly lower under the trees as compared to positions 1 and $3 \mathrm{~m}$ away from tree rows. Maize grain and straw yields as well as nutrient accumulation were higher under the trees and decreased with increasing the distance from the trees.
\end{abstract}

Index terms: semi-arid, light fraction of soil organic matter, soil moisture.

\section{INTRODUÇÃO}

A sustentabilidade de sistemas agrícolas familiares não irrigados no Agreste da Paraíba, assim como no restante do semi-árido nordestino, é um dos aspectos atuais mais relevantes para a região. Entretanto, o cultivo agrícola e a retirada dos produtos agrícolas após a colheita, sem adoção de práticas que visem à reposição de nutrientes, podem causar a degradação das propriedades físicas, químicas e biológicas dos solos (Salcedo et al., 1997; Menezes \& Sampaio, 2000; ASPTA, 2000). Na região semi-árida, estes efeitos são mais intensos em razão da elevada quantidade de energia radiante (13-19 $\left.\mathrm{MJ} \mathrm{m}^{-2} \mathrm{dia}^{-1}\right)$, da menor variação do comprimento do dia e das elevadas temperaturas que, associadas com a disponibilidade de água durante a época chuvosa do ano, resultam em altas taxas de oxidação dos restos vegetais e da matéria orgânica do solo (Frainderaich, 2000; Mielniczuk et al., 2003). Isto se verifica principalmente em áreas recém-desmatadas, submetidas a cultivo itinerante e intensivo, onde a acelerada mineralização da matéria orgânica leva a perdas da produtividade vegetal (Tiessen et al., 1992; Salcedo et al., 1997). Esses fatos, associados à ocorrência de chuvas de alta intensidade, exigem práticas de manejo do solo que priorizem a cobertura permanente e a manutenção da matéria orgânica.

Estudos recentes têm demonstrado que a preservação de árvores durante o corte da caatinga ou o plantio de espécies nativas ou introduzidas podem contribuir para a preservação ou recuperação da fertilidade do solo em campos agrícolas ou pastagens no semi-árido nordestino. Segundo Araújo Filho (1990), em sistemas silvopastoris em áreas de caatinga, a preservação de 30 \% das árvores durante o corte da vegetação nativa incrementou a produção de forragem e carne em comparação com áreas onde todas as árvores foram removidas. Menezes \& Salcedo (1999) e Menezes et al. (2002) encontraram maiores teores de matéria orgânica e nutrientes $(\mathrm{N}, \mathrm{P}, \mathrm{Ca}$, $\mathrm{Mg}$ ) em amostras de solo coletadas debaixo das copas de Ziziphus joazeiro e Prosopis juliflora do que nas áreas ao redor das árvores cultivadas com capim-buffel 
(Cenchrus ciliaris). Estes estudos demonstraram que sistemas em que são consorciadas árvores resistentes à seca com culturas agrícolas ou pastagens podem aumentar a produção de biomassa e manter a fertilidade do solo no semi-árido nordestino (Wick et al., 2000; Menezes et al., 2002). Entretanto, a importância e a efetividade de cada um destes processos dependem das condições específicas de cada ecossistema e das práticas de manejo adotadas.

Gliricidia sepium é uma leguminosa que tem sido plantada em propriedades rurais do Agreste paraibano graças à sua alta capacidade de produzir biomassa em condições de baixa disponibilidade hídrica. Esta espécie geralmente é plantada em cercas-vivas nas propriedades e, recentemente, tem sido plantada nos campos de cultivo, em fileiras suficientemente espaçadas entre si, para permitir o plantio de culturas agrícolas ou forrageiras entre elas. $\mathrm{O}$ manejo da gliricídia neste sistema é feito por cortes periódicos da copa das árvores (geralmente de dois a três cortes por ano). Estudos prévios demonstram que a introdução da gliricídia em campos de cultivo agrícola pode apresentar várias vantagens potenciais, a saber: produção de biomassa rica em nutrientes para adubação orgânica, presença de um sistema radicular perene, cobertura e proteção do solo, manutenção ou melhoria das condições físicas, químicas e biológicas do solo, manutenção da microfauna em profundidade e produção de forragem para alimentação animal, além de outros produtos florestais ou não-florestais (Schroth \& Lehmann, 1995; Gómez \& Preston, 1996; Esquivel et al., 1998; Barreto \& Fernandes, 2001).

Na região do Agreste da Paraíba, são praticamente inexistentes informações quanto ao impacto da introdução de espécies arbóreas em campos de cultivo agrícola. Sendo assim, delineou-se o presente estudo com o objetivo geral de avaliar a produção e composição química do milho, alterações em características do solo e microclima, e a produção de folhedo a diferentes distâncias de plantas de Gliricidia sepium.

\section{MATERIAL E MÉTODOS}

\section{Descrição da área de estudo}

Os estudos foram desenvolvidos no período de outubro de 2002 a julho de 2004, no Centro Agroecológico São Miguel (CASM), sede da ONG Assessoria e Serviços a Projetos de Agricultura Alternativa (ASPTA), no município Esperança, no Agreste paraibano. O CASM está localizado geograficamente a $7^{\circ} 19^{\prime}$ de latitude sul e $33^{\circ} 51^{\prime}$ de longitude oeste, a uma altitude de $635 \mathrm{~m}$. O clima da região caracteriza-se por apresentar uma estação chuvosa, de março a agosto, e uma estação seca, de setembro a fevereiro. A precipitação pluviométrica média anual é de $800 \mathrm{~mm}$, e os totais anuais em, 2003 e 2004, atingiram 653 e $1.000 \mathrm{~mm}$, respectivamente. O solo na área experimental é classificado como um Neossolo regolítico (Embrapa, 1999a), com declividade em torno de $0,05 \mathrm{~m} \mathrm{~m}^{-1}$, textura franco-arenosa, apresentando, na camada de $0-20 \mathrm{~cm}, 830 \mathrm{~g} \mathrm{~kg}^{-1} \mathrm{de}$ areia, $60 \mathrm{~g} \mathrm{~kg}^{-1}$ de argila e $110 \mathrm{~g} \mathrm{~kg}^{-1}$ de silte.

Em uma área de aproximadamente 0,5 ha, foram estabelecidas, em 1996, fileiras de G. sepium no espaçamento de $6 \mathrm{~m}$ si e $1 \mathrm{~m}$ entre as plantas. De 1996 a 2002, a área entre as linhas de árvores foi cultivada anualmente com batata. Em outubro de 2002, em pontos distribuídos ao acaso dentro da área experimental, foram estabelecidas quatro parcelas, com dimensão de 6 x 8 m cada uma. Em 2003 e 2004, as aléias de $G$. sepium foram podadas duas vezes por ano, a uma altura de $1 \mathrm{~m}$. O primeiro corte foi realizado no início da estação chuvosa (fevereiro a março), o segundo quatro meses após o primeiro (final de julho). A biomassa cortada foi retirada e utilizada como forragem.

\section{Coleta inicial de amostras de solo e análises químicas}

$\mathrm{Na}$ parte central de cada parcela, foram estabelecidas três posições de amostragem em transetos perpendiculares às fileiras de árvores: (a) na fileira de árvores (0 m); (b) a $1 \mathrm{~m}$ da fileira; e (c) a $3 \mathrm{~m}$ da fileira. Em novembro de 2002 , em cada posição de amostragem ( 0 , 1 e $3 \mathrm{~m}$ ), foram coletadas dez amostras de solo na profundidade de $0-10 \mathrm{~cm}$. As amostras foram misturadas, formando uma amostra composta, levadas ao laboratório, secas ao ar, passadas em peneiras de $2 \mathrm{~mm}$ e analisadas quanto ao $\mathrm{pH}$, medido em água na relação 1:2,5, $\mathrm{P}$ disponível e $\mathrm{K}$ trocável extraídos com Mehlich-1 de acordo com os métodos descritos pela Embrapa (1999b), e carbono orgânico total, pelo método de Snyder \& Trofymow (1984).

\section{Quantificação da queda de folhedo e da matéria orgânica leve-livre (MOL) do solo}

Em 2003, foi avaliada a dinâmica da queda de folhedo e da MOL do solo. Para a coleta do folhedo caído das árvores de G. sepium, foram utilizadas cestas com diâmetro de $35 \mathrm{~cm}$, confeccionadas com telas de náilon de abertura de $1 \mathrm{~mm}$, instaladas a uma altura de $20 \mathrm{~cm}$ do solo. Foram colocadas oito cestas, por parcela, em dois transetos perpendiculares às fileiras de $G$. sepium a distâncias de 0, 1, 2 e $3 \mathrm{~m}$ das fileiras. O folhedo foi coletado das cestas, quinzenalmente, seco em estufa até massa constante e analisado para determinação do teor de nutrientes por meio dos métodos descritos pela Embrapa (1999b). Para quantificar a dinâmica da MOL do solo, foram realizadas coletas de solo em quatro épocas do ano: $1^{\mathrm{a}}$ amostragem - plena estação das chuvas (abril); $2^{\text {a }}$ amostragem - final das chuvas (julho); $3^{\mathrm{a}}$ amostragem - plena seca (outubro); $4^{\mathrm{a}}$ amostragem - final da seca (janeiro/2004). As amostras de solo para quantificar a MOL foram coletadas nas mesmas posições de amostragem nas parcelas, dos transetos 
antes descritos, na profundidade de $0-10 \mathrm{~cm}$. Subamostras do solo foram levadas ao laboratório do Departamento de Solos da Universidade Federal da Paraíba (UFPB) para determinação da MOL pela flotação em água, utilizando o método densimétrico (Gregorich \& Ellert, 1993; Fraga \& Salcedo, 2004). Os teores de $\mathrm{CO}$ e nutrientes da MOL do solo não foram determinados, uma vez que as quantidades de MOL extraídas não foram suficientes.

\section{Condições microclimáticas}

A umidade do solo $(0-20 \mathrm{~cm})$ foi medida, semanalmente, ao longo das estações de cultivo (março a agosto), em 2003 e 2004, utilizando-se uma sonda de nêutrons. Em cada posição de amostragem, foi instalado um tubo de acesso de $\mathrm{Al}$. A relação entre a leitura da sonda, em contagens por minuto (cpm), e o conteúdo de água $(\theta)$ do solo, foi estabelecida nas estações úmida e seca, conforme descrito por IAEA (2003). Para isto, foram coletadas amostras de solo no entorno de cada tubo, para determinar $\theta$ pelo metodo gravimétrico. Para evitar efeitos eletrônicos derivados da temperatura e outros fatores que pudessem interferir na sonda, não foram usados diretamente os dados de cpm obtidos em campo, mas, sim, a relação de contagem relativa $(\mathrm{CR})$, segundo descrito por IAEA (2003).

A temperatura do solo foi determinada usando sensores automáticos HOBO, com capacidade de armazenamento de dados, colocados em posição vertical, na profundidade de 5 a $10 \mathrm{~cm}$. As medidas foram realizadas simultaneamente nas posições de 0 a $3 \mathrm{~m}$ de distância das árvores em uma das parcelas experimentais. Os sensores foram envolvidos com filme plástico fino para evitar danos pela umidade do solo e foram programados para realizar medidas de temperatura a cada 30 min por um período de 270 dias.

Em uma das parcelas, foi medida a temperatura do ar, nas posições a 0 e $3 \mathrm{~m}$ de distância das árvores, usando sensores HOBO colocados dentro de pequenas caixas de madeira com persianas nas laterais, para permitir ventilação, e fixadas a um poste de madeira $1 \mathrm{~m}$ acima da superfície do solo. As caixas foram posicionadas próximas (1 m de distância) aos sensores enterrados para medir a temperatura do solo.

\section{Produção de milho}

Em 2003 e 2004, cada parcela foi cultivada com milho (Zea mays L.) variedade "pontinha", obtido no banco de sementes da ASPTA. Foram semeadas duas sementes por cova, em espaçamento de 1 x 0,20 m. Duas semanas após a emergência das plântulas, realizou-se o desbaste, deixando uma planta por cova (50.000 plantas ha-1). O controle de plantas espontâneas foi realizado com enxada 15 a 20 dias após a semeadura. Na ocasião da colheita do milho,
120 dias após a semeadura, foram realizadas avaliações da produtividade de palha e de grãos do milho (12 \% de umidade) de acordo com as distâncias das fileiras de árvores (1, 2 e $3 \mathrm{~m}$ ). As amostras de material vegetal foram secas em estufa, pesadas para determinar o teor de matéria seca e moídas (Moinho tipo Wiley) para análises químicas. As determinações de N, P e K na palha e grãos do milho foram realizadas de acordo com os métodos descritos pela Embrapa (1999b).

\section{Análises estatísticas}

O desenho experimental utilizado foi em blocos casualizados, com quatro repetições, em que os tratamentos foram as posições de amostragem a distâncias iguais a 0,1 e $3 \mathrm{~m}$ das fileiras de árvores. Os valores da concentração de nutrientes e umidade do solo, queda de folhedo, produtividade e nutrientes do milho nas posições de amostragem foram submetidos à análise de variância utilizando o programa SAS Statistical Package (SAS, 1995), e as médias comparadas pelo teste de Tukey $(\mathrm{P}<0,05)$. Os dados de temperatura do solo e do ar foram submetidos a análises de estatística descritiva.

\section{RESULTADOS E DISCUSSÃO}

\section{Queda de folhedo}

A massa seca de folhedo debaixo das árvores atingiu $1.390 \mathrm{~kg} \mathrm{ha}{ }^{-1}$ com diminuição gradual até valores de $270 \mathrm{~kg} \mathrm{ha}^{-1}$ na distância de $3 \mathrm{~m}$ das árvores (Quadro 1), somando-se todo o folhedo caído durante o período de queda, de julho a novembro de 2003. Estes valores são baixos, quando comparados aos resultados de outros estudos realizados em florestas tropicais sobre solos de média a baixa fertilidade, cujos valores médios foram de 11 e $9 \mathrm{Mg} \mathrm{ha}^{-1}$ ano $^{-1}$ de folheado caído, respectivamente (Vistousek \& Sanford, 1986).

No presente estudo, as quantidades de N, P e K aportadas via queda de folhedo embaixo das árvores foram estimadas em 27,8, 1,39 e $5 \mathrm{~kg} \mathrm{ha}^{-1}$, respectivamente (Quadro 1), diminuindo para 5,4, 0,27 e $2,7 \mathrm{~kg} \mathrm{ha}^{-1}$ de $\mathrm{N}, \mathrm{P}$ e $\mathrm{K}$ na distância de $3 \mathrm{~m}$ das fileiras de G. sepium.

A queda de folhedo observada neste estudo, embora tenha sido baixa comparada à de ecossistemas tropicais úmidos, foi comparável àquela encontrada em ecossistemas de caatinga arbórea e savana. Dantas (2003) realizou um estudo em uma caatinga arbórea no Agreste paraibano e quantificou uma queda de folhedo anual de 1,5 $\mathrm{Mg} \mathrm{ha}^{-1}$, em média, ao longo de dois anos do estudo. Os baixos valores observados no presente estudo são, em parte, resultantes da baixa densidade de plantio e da baixa precipitação pluviométrica da região do estudo. Além disso, o 
Quadro 1. Massa seca e conteúdo de $\mathrm{N}, \mathrm{P}$ e $\mathrm{K}$ no folhedo caído no período de julho a novembro de 2003 a diferentes distâncias das árvores de G. sepium plantadas em fileiras com espaçamento de $6 \mathrm{~m}$ entre fileiras e $1 \mathrm{~m}$ entre árvores, consorciadas com milho em um Neossolo Regolítico, no município de Esperança (PB)

\begin{tabular}{lllll}
\hline $\begin{array}{c}\text { Distância da a } \\
\text { án }\end{array}$ & Massa seca & N & P & K \\
\hline
\end{tabular}

\begin{tabular}{ccccl}
$\mathrm{m}$ & \multicolumn{5}{c}{$\mathrm{kg} \mathrm{ha}-1$} \\
\cline { 2 - 5 } 0 & $1.390 \mathrm{a}^{(1)}$ & $27,8 \mathrm{a}$ & $1,39 \mathrm{a}$ & $5 \mathrm{a}$ \\
1 & $970 \mathrm{a}$ & $19,4 \mathrm{a}$ & $0,97 \mathrm{a}$ & $3,49 \mathrm{a}$ \\
2 & $360 \mathrm{~b}$ & $7,2 \mathrm{~b}$ & $0,36 \mathrm{~b}$ & $1,30 \mathrm{~b}$ \\
3 & $270 \mathrm{~b}$ & $5,4 \mathrm{~b}$ & $0,27 \mathrm{~b}$ & $0,97 \mathrm{~b}$ \\
Média & 748 & 15 & 0,75 & 2,70
\end{tabular}

(1) Médias, na coluna, seguidas pela mesma letra não diferem estatisticamente pelo teste de Tukey $(\mathrm{P}<0,05)$.

cultivo sucessivo do solo associado à retirada contínua da produção vegetal, sem a reposição dos restos vegetais ou outra fonte externa de insumos, favorece a degradação das propriedades físicas, químicas e biológicas do solo. Nestas condições, é provável que a demanda nutricional das plantas não seja satisfeita pelo processo de ciclagem de nutrientes, limitando, assim, a produtividade de biomassa e a quantidade de folhedo que cai ao solo anualmente.

\section{Carbono orgânico total (COT), P, K e matéria orgânica leve (MOL) do solo}

Sete anos após o plantio das árvores de G. sepium, os teores de COT do solo não apresentaram diferenças significativas ao longo dos transetos de amostragem (Quadro 2). Alguns estudos têm demonstrado que, em sistemas agroflorestais, as quantificações das mudanças ocorridas em algumas variáveis no solo, como COT total, não devem ser feitas precocemente, visto que é considerado improvável a percepção dessas modificações em um prazo inferior a 10 anos (Barrios et al., 1997; Buresh \& Tian, 1998; Esquivel et al., 1998; Murage et al., 2000; Etchevers et al., 2000; Mendonça et al., 2001, Barreto \& Fernandes, 2001). Além disso, as práticas de cultivo sucessivo, especialmente pela intensidade de revolvimento do solo dentro das aléias durante os sete anos desde o plantio das árvores, não favoreceram o acúmulo maior de COT em razão da influência que esta prática apresenta sobre a temperatura, umidade, aeração do solo e da maior exposição da matéria orgânica do solo (MOS) à degradação (Barreto \& Fernandes, 2001). Numa escala mais pontual, destaca-se a baixa quantidade de folhedo que cai da parte aérea e que irá formar a matéria orgânica, uma vez que o acúmulo de matéria orgânica no solo é regulado pela quantidade de resíduos orgânicos que caem da parte aérea das plantas e por sua taxa de decomposição. Quanto maior a quantidade que cai desse material e quanto menor sua velocidade de decomposição, maior o teor de COT no solo (Correia \& Andrade, 1999). Por isso, nestas condições de cultivo, a matéria orgânica total do solo não foi um indicador adequado para apontar mudanças de manejo no período entre o plantio das árvores e a amostragem do solo.

Por outro lado, os teores de matéria orgânica leve (MOL) variaram significativamente, tanto em relação às distâncias das árvores como nas distintas épocas de amostragem. Em geral, a MOL foi maior embaixo da copa das árvores do que a 1 e $3 \mathrm{~m}$ de distância, ao longo das quatro épocas de amostragem e transetos estudados (Quadro 3). O menor valor da MOL a $0 \mathrm{~m}$ foi encontrado na plena estação chuvosa, com média de $2,15 \mathrm{~g} \mathrm{~kg}^{-1}$ e o maior valor no final da seca, com média de $3,72 \mathrm{~g} \mathrm{~kg}^{-1}$. O aumento de MOL na época mais seca deveu-se à característica caducifólia da G. sepium. A 1 e $3 \mathrm{~m}$ de distância das árvores, não ocorreram diferenças significativas, e isso pode ser explicado pelo cultivo sucessivo entre as fileiras de árvores e pela baixa deposição de folhedo nesses pontos de amostragem. Ao final da seca (janeiro), a MOL quantificada a 0 m foi 73,43 e $36 \%$ maior do que nas épocas de estação chuvosa (abril), final da estação chuvosa (julho) e plena seca (outubro), sustentando a idéia que sua quantidade no solo e composição apresentam variabilidade espacial e sazonal em relação a outras frações de matéria orgânica do solo, o que mostra ser um bom indicador capaz de detectar mudanças no manejo da cobertura vegetal nesse sistema.

Os teores de K e de $\mathrm{P}$ extraíveis do solo (Quadro 2) tiveram comportamento similar aos teores da MOL (Quadro 3), sendo mais elevados a $0 \mathrm{~m}$ do que a $1 \mathrm{e}$ $3 \mathrm{~m}$ de distância das árvores. $\mathrm{O}$ teor de $\mathrm{P}$ a $0 \mathrm{~m}$ não

Quadro 2. Teores médios de carbono orgânico total (COT), P, K e pH do solo em amostras coletadas na profundidade de $0-10 \mathrm{~cm}$ a 0,1 e $3 \mathrm{~m}$ de distância de árvores de G. sepium, sete anos após o seu plantio em um Neossolo Regolítico, no município de Esperança (PB)

\begin{tabular}{ccccc}
\hline $\begin{array}{c}\text { Distância da } \\
\text { árvore }\end{array}$ & COT & P & K & $\mathbf{p H}$ \\
\hline $\mathrm{m}$ & $\mathrm{g} \mathrm{kg}^{-1}$ & \multicolumn{2}{c}{$-\mathrm{mg} \mathrm{kg}^{-1}-$} \\
0 & $9,7 \mathrm{a}^{(1)}$ & $8,6 \mathrm{a}$ & $376 \mathrm{a}$ & $6,6 \mathrm{a}$ \\
1 & $8,9 \mathrm{a}$ & $7,5 \mathrm{ab}$ & $178 \mathrm{~b}$ & $6,4 \mathrm{a}$ \\
3 & $8,4 \mathrm{a}$ & $6,1 \mathrm{~b}$ & $162 \mathrm{~b}$ & $6,4 \mathrm{a}$
\end{tabular}

$\overline{{ }^{(1)} \text { Médias, na linha, seguidas pela mesma letra não diferem }}$ estatisticamente pelo teste de Tukey $(\mathrm{P}<0,05)$. 
foi significativamente diferente do teor a $1 \mathrm{~m}$, porém foi significativamente maior do que a $3 \mathrm{~m}$ de distância das árvores. Já os teores de K foram 2,1 e 2,3 vezes maiores a $0 \mathrm{~m}$ que os teores a 1 e $3 \mathrm{~m}$, respectivamente. A maior concentração de nutrientes do solo embaixo das árvores é explicada pela deposição de folhas de G. sepium, aliada à sua composição química, rica em $\mathrm{N}, \mathrm{P}, \mathrm{Ca}, \mathrm{Mg}$ e, principalmente, $\mathrm{K}$ (Nair, 1995; Kwesiga, 1994, Gomez \& Preston 1996).

\section{Umidade do solo}

A umidade do solo (0 a $20 \mathrm{~cm}$ ), avaliada nos períodos de março a agosto de 2003 e 2004, foi significativamente menor nas fileiras de G. sepium (0 m) em comparação com a umidade a $3 \mathrm{~m}$ de distância das fileiras. Entre 0 e $1 \mathrm{~m}$, a umidade do solo não apresentou diferenças significativas. Em 2003 e 2004, a umidade do solo a 3 m de distância das árvores nos meses monitorados foi, em média, de 20 e $17 \%$ maior em relação a 0 e $1 \mathrm{~m}$, respectivamente (Quadro 4). Tais resultados revelam que a presença da $G$. sepium leva a uma maior absorção de água nas posições próximas às fileiras de árvores, ou seja, as raízes laterais das árvores nesse sistema aparentemente são capazes de capturar significativamente mais água a distâncias pelo menos cerca de $1 \mathrm{~m}$, mas menores que $3 \mathrm{~m}$ das fileiras (Quadro 4). É também possível que a menor umidade do solo embaixo das árvores seja, em parte, resultante da interceptação pela copa de uma fração da água precipitada. Essa água interceptada pode ficar parcialmente retida nas folhas e escorrer pelos galhos das árvores e infiltrarse no solo imediatamente adjacente ao caule das árvores. Entretanto, não há dados disponíveis sobre a interceptação de chuva pela copa das árvores nesses sistemas no Nordeste do Brasil.

Quadro 3. Matéria orgânica leve do solo (MOL) na profundidade de $\mathbf{0}-10 \mathrm{~cm}$ em quatro épocas do ano (de abril 2003 a janeiro de 2004) dentro de um sistema agroflorestal com $G$. sepium ao longo de um transeto de amostragem partindo debaixo da copa das árvores $(0 \mathrm{~m})$ até o centro das parcelas (3 m), no município de Esperança (PB)

\section{Distância da árvore}

\begin{tabular}{ll} 
Época (meses) \\
\hline Abril Julho Outubro Janeiro
\end{tabular}

$\mathrm{m}$

0

1

3

\begin{tabular}{|c|c|c|c|}
\hline $2,15 \mathrm{Ca}^{(1)}$ & $2,61 \mathrm{Ba}$ & $2,73 \mathrm{Ba}$ & $3,72 \mathrm{Aa}$ \\
\hline $1,04 \mathrm{Ab}$ & $1,09 \mathrm{Ab}$ & $1,10 \mathrm{Ab}$ & $1,24 \mathrm{Ab}$ \\
\hline $0,95 \mathrm{Bb}$ & $0,85 \mathrm{Bb}$ & $0,88 \mathrm{Bb}$ & $1,20 \mathrm{Ab}$ \\
\hline
\end{tabular}

(1) Médias, nas linhas, seguidas pela mesma letra maiúscula e, médias, nas colunas, seguidas pela mesma letra minúscula não diferem estatisticamente pelo teste de Tukey $(\mathrm{P}<0,05)$.
Quadro 4. Conteúdo médio de água no solo $(0-20 \mathrm{~cm})$ a diferentes distâncias de árvores de G. sepium plantadas em fileiras espaçadas $6 \mathrm{~m}$ entre si e consorciadas com milho em um Neossolo Regolítico, no município de Esperança (PB), no período de março a agosto de 2003 e 2004

\begin{tabular}{lccc}
\hline \multirow{4}{*}{ Mês } & Distância em relação às fileiras de $\boldsymbol{G}$. sepium \\
\cline { 2 - 4 } & $\mathbf{0} \mathbf{~ m}$ & $\mathbf{1} \mathbf{~ m}$ \\
\hline \multicolumn{4}{c}{ Conteúdo de água no solo, $\mathrm{cm}^{3} \mathrm{~cm}^{-3}$} \\
Março & $0,131 \mathrm{~b}(1)$ & $0,135 \mathrm{~b}$ & $0,150 \mathrm{a}$ \\
Abril & $0,127 \mathrm{~b}$ & $0,131 \mathrm{~b}$ & $0,151 \mathrm{a}$ \\
Maio & $0,112 \mathrm{~b}$ & $0,113 \mathrm{~b}$ & $0,142 \mathrm{a}$ \\
Junho & $0,121 \mathrm{~b}$ & $0,121 \mathrm{~b}$ & $0,145 \mathrm{a}$ \\
Julho & $0,120 \mathrm{~b}$ & $0,126 \mathrm{~b}$ & $0,145 \mathrm{a}$ \\
Agosto & $0,121 \mathrm{~b}$ & $0,121 \mathrm{~b}$ & $0,144 \mathrm{a}$ \\
& & 2004 & \\
Marco & $0,149 \mathrm{~b}$ & $0,176 \mathrm{~b}$ & $0,219 \mathrm{a}$ \\
Abril & $0,118 \mathrm{~b}$ & $0,126 \mathrm{~b}$ & $0,161 \mathrm{a}$ \\
Maio & $0,133 \mathrm{~b}$ & $0,137 \mathrm{~b}$ & $0,179 \mathrm{a}$ \\
Junho & $0,141 \mathrm{~b}$ & $0,137 \mathrm{~b}$ & $0,179 \mathrm{a}$ \\
Julho & $\mathrm{SD}(2)$ & $\mathrm{SD}$ & $\mathrm{SD}$ \\
Agosto & $0,100 \mathrm{~b}$ & $0,101 \mathrm{~b}$ & $0,126 \mathrm{a}$ \\
\hline
\end{tabular}

(1) Médias, na linha, seguidas pela mesma letra não diferem estatisticamente pelo teste de Tukey $(\mathrm{P}<0,05) .{ }^{(2)} \mathrm{SD}=\mathrm{Sem}$ dados.

\section{Condições microclimáticas}

O plantio de árvores de G. sepium dentro das áreas cultivadas influenciou a temperatura do ar e do solo, principalmente nos meses correspondentes às épocas de plena estação chuvosa e final das chuvas, ou seja, maio e julho, respectivamente (Quadro 5). As temperaturas mínimas do ar foram similares nas posições embaixo das árvores e a $3 \mathrm{~m}$ de distância; entretanto, as temperaturas máximas no período de estudo foram, em média, $2^{\circ} \mathrm{C}$ mais elevadas a $3 \mathrm{~m}$ do que embaixo da copa das árvores. Esta influência foi observada principalmente durante os meses de plena estação chuvosa (maio a junho), ou seja, durante o período de crescimento das copas das árvores após a poda. Já na plena seca e no final da seca (outubro a janeiro), não foram observadas diferenças entre as temperaturas máximas do ar em ambas posições de amostragem. Provavelmente, isto aconteceu por causa da poda das árvores no final das chuvas (julho) e da característica caducifólia da G. sepium, que permanece sem folhas durante os períodos de maior estresse hídrico. A poda realizada ao final das chuvas fez com que as copas das árvores permanecessem 
bastante reduzidas durante e estação seca, com menor capacidade de sombreamento. Na estação chuvosa, as árvores após a poda tiveram uma rápida reposição das suas folhas e, conseqüentemente, maior capacidade de sombreamento.

Resultados similares foram observados quanto às temperaturas mínimas e máximas do solo (Quadro 5). As temperaturas mínimas foram similares a 0 e $3 \mathrm{~m}$ de distâncias das árvores. Entretanto as temperaturas máximas do solo foram inferiores a $0 \mathrm{~m}$, ao longo das quatro épocas do ano estudadas, apresentando, em geral, valores entre 24 e $31^{\circ} \mathrm{C}$, enquanto a $3 \mathrm{~m}$ a variação das temperaturas máximas no solo oscilaram entre 27 e $41^{\circ} \mathrm{C}$ (Quadro 5). Ao longo do ano, a temperatura máxima do solo a $3 \mathrm{~m}$ de distância das árvores foi, em média, $6{ }^{\circ} \mathrm{C}$ mais alta do que debaixo da copa das árvores.

Dados semelhantes sobre o microclima dentro de diversos sistemas de manejo agroflorestal e variadas espécies arbóreas foram relatados em outros estudos. Menezes et al. (2002) encontraram que as temperaturas máximas do ar e do solo foram, em média, 2 e $16{ }^{\circ} \mathrm{C}$ mais baixas debaixo das copas de Ziziphus joazeiro em relação a áreas com Cenchrus ciliaris. Outro estudo realizado por Sierra et al. (2002) mostrou que a temperatura do solo num sistema silvopastoril foi, em média, $2^{\circ} \mathrm{C}$ menor do que em áreas com pastagem. Os resultados desses trabalhos demonstram que a introdução de espécies arbóreas dentro de sistemas agrícolas pode exercer uma ação reguladora sobre microclima, como observado no presente estudo. Os efeitos das espécies arbóreas sobre a temperatura do ar e do solo são parâmetros importantes no desenho dos sistemas agroflorestais, uma vez que as inter-relações de fotossíntese e respiração dependem grandemente da temperatura.
Na região semi-árida do Nordeste do Brasil, a quantidade de energia radiante e a temperatura são elevadas e uniformemente distribuídas ao longo do ano. Dessa maneira, em geral, esses dois fatores não limitam a fotossíntese; todavia, valores elevados de temperatura podem levar a perdas excessivas de carbono por meio do processo respiratório, bem como a perdas de água dos sistemas (Moreira \& Siqueira, 2002).

\section{Produção de grãos e palha do milho}

A produção de grãos e de palha do milho observada no presente estudo foi relativamente baixa, levando em consideração as médias da região semi-árida, que se situam em torno de 700 e $2.000 \mathrm{~kg} \mathrm{ha}^{-1}$ (Menezes \& Sampaio, 2000). Verificou-se que a produção do milho acompanhou o gradiente de fertilidade observado nas análises químicas de solo. Em 2003, a produção de grãos foi de $476 \mathrm{~kg} \mathrm{ha}^{-1}$ a $1 \mathrm{~m}$ de distância das árvores, com uma diminuição gradual até $399 \mathrm{~kg}^{-1}$ a $3 \mathrm{~m}$ das árvores (Quadro 6). Em 2004, a produção média de grãos a $1 \mathrm{~m}$ das árvores foi de $318 \mathrm{~kg} \mathrm{ha}^{-1}$, com uma diminuição gradual até $122 \mathrm{~kg} \mathrm{ha}^{-1}$ a $3 \mathrm{~m}$ das árvores (Quadro 6), valores 34 e 69 \% menores, em relação a 2003. Comportamento similar foi observado para ambos os anos quanto à produção de palha (Quadro 6).

A quantidade de N, P e K absorvido pelo milho foi maior nas fileiras próximas às árvores de $G$. sepium. Em 2003, as quantidades absorvidas de N, P e K (grão + palha) a $1 \mathrm{~m}$ de distância das árvores foram de 15 , 4 e $19 \mathrm{~kg} \mathrm{ha}^{-1}$, respectivamente. Nas fileiras de milho a $3 \mathrm{~m}$ de distância das árvores, as quantidades de $\mathrm{N}$, $\mathrm{P}$ e $\mathrm{K}$ absorvidas foram de 10,3 e $11 \mathrm{~kg} \mathrm{ha}^{-1}$, respectivamente (Quadro 6). Resultados similares foram observados em 2004, porém, com menores

Quadro 5. Médias mensais das temperaturas máximas e mínimas do ar e do solo (0-10 cm) ao longo do ano embaixo e a $3 \mathrm{~m}$ de distância de fileiras de $G$. sepium consorciada com milho em um sistema agroflorestal no município de Esperança (PB)

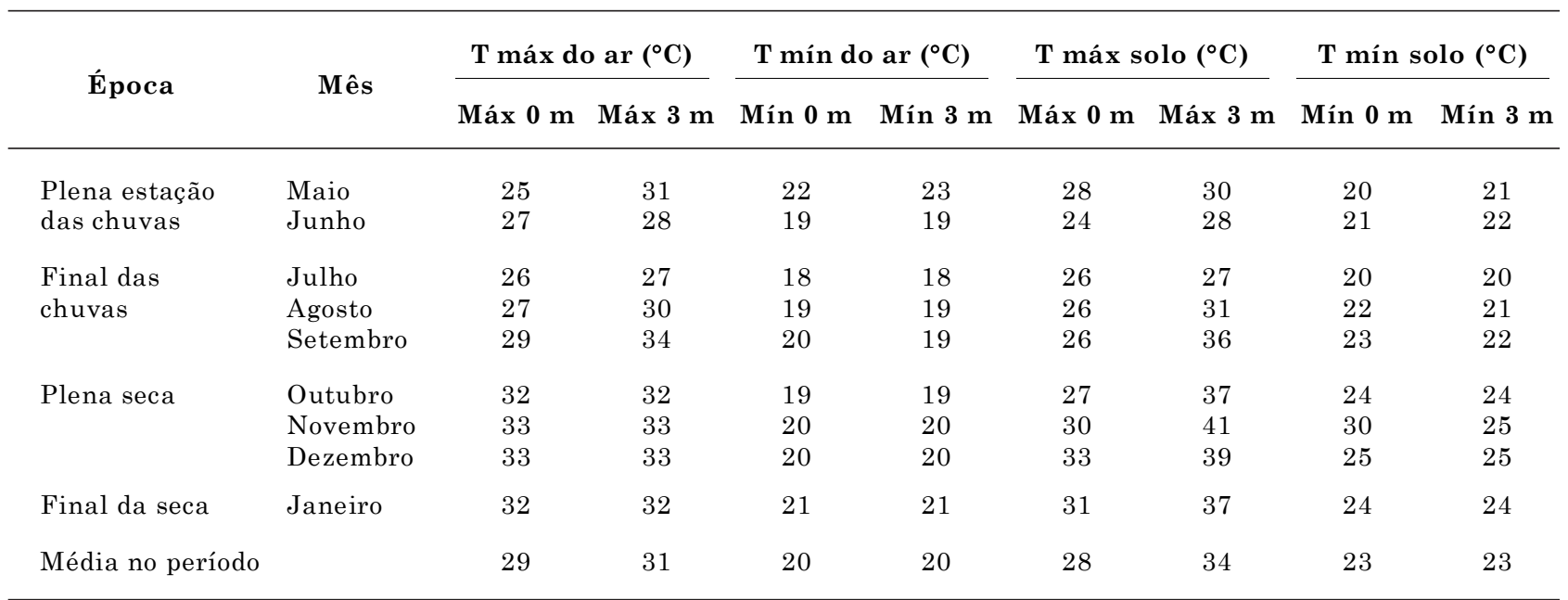


Quadro 6. Produção e composição química de grãos e de palha do milho semeado a diferentes distâncias de fileiras de G. sepium em um sistema agroflorestal de cultivo no município de Esperança (PB)

\begin{tabular}{|c|c|c|c|c|c|c|c|c|}
\hline \multirow{2}{*}{$\begin{array}{c}\text { Distância da } \\
\text { árvore }\end{array}$} & \multicolumn{4}{|c|}{ Grão } & \multicolumn{4}{|c|}{ Palha } \\
\hline & MS & $\mathbf{N}$ & $\mathbf{P}$ & $\mathbf{K}$ & MS & $\mathbf{N}$ & $\mathbf{P}$ & $\mathbf{K}$ \\
\hline \multirow[t]{2}{*}{$\mathrm{m}$} & \multicolumn{8}{|c|}{$-\mathrm{kg} \mathrm{ha}^{-1}$} \\
\hline & \multicolumn{8}{|c|}{2003} \\
\hline 1 & $479 \mathrm{a}^{(1)}$ & $7,18 \mathrm{a}$ & $2,35 \mathrm{a}$ & $3,02 \mathrm{a}$ & $1156 \mathrm{a}$ & 8,09 a & $2,08 \mathrm{a}$ & $16,41 \mathrm{a}$ \\
\hline 2 & $340 \mathrm{a}$ & $5,10 \mathrm{a}$ & $1,66 \mathrm{a}$ & $2,14 \mathrm{a}$ & $1106 \mathrm{a}$ & $7,74 \mathrm{a}$ & $1,99 \mathrm{a}$ & $15,70 \mathrm{a}$ \\
\hline \multirow[t]{2}{*}{3} & 399 a & $5,98 \mathrm{a}$ & $2,05 \mathrm{a}$ & $2,51 \mathrm{a}$ & $629 \mathrm{~b}$ & $4,40 \mathrm{~b}$ & $1,13 \mathrm{~b}$ & $8,93 \mathrm{~b}$ \\
\hline & \multicolumn{8}{|c|}{2004} \\
\hline 1 & $317 \mathrm{a}$ & $4,75 \mathrm{a}$ & $1,55 \mathrm{a}$ & $1,99 \mathrm{a}$ & $1138 \mathrm{a}$ & $7,96 \mathrm{a}$ & $2,04 \mathrm{a}$ & $16,15 \mathrm{a}$ \\
\hline 2 & $179 \mathrm{~b}$ & $2,68 \mathrm{~b}$ & $0,88 \mathrm{~b}$ & $1,13 \mathrm{~b}$ & $826 \mathrm{~b}$ & $5,78 \mathrm{~b}$ & $1,48 \mathrm{~b}$ & $11,72 \mathrm{~b}$ \\
\hline 3 & $122 \mathrm{c}$ & $1,83 \mathrm{c}$ & $0,59 \mathrm{c}$ & $0,77 \mathrm{c}$ & $809 \mathrm{~b}$ & $5,66 \mathrm{~b}$ & $1,46 \mathrm{~b}$ & $11,49 \mathrm{~b}$ \\
\hline
\end{tabular}

(1) Médias, na coluna, seguidas pela mesma letra não diferem estatisticamente pelo teste de Tukey $(\mathrm{P}<0,05)$.

quantidades absorvidas, em decorrência da menor produção nesse ano.

As baixas produções de grãos e palha do milho nos dois anos do estudo estão relacionadas com a baixa fertilidade do solo, já que estas parcelas foram cultivadas sem nenhum tipo de adubação orgânica ou química durante os sete anos anteriores ao presente estudo. Além disso, neste estudo, tanto os grãos e palha do milho, quanto a biomassa da parte área da G. sepium, foram retirados após cada colheita ou poda, o que contribuiu para diminuição do estoque de nutrientes do sistema. A palha do milho e a biomassa da G. sepium são normalmente utilizadas como forragem para animais externos ao sistema, enquanto o esterco desses animais não retorna aos campos de cultivo do milho. Uma vez que as árvores são podadas anualmente, o processo de ciclagem de nutrientes via queda de folhedo é insuficiente para atender à demanda nutricional do milho (Quadro 1). No geral, o balanço de nutrientes entre as entradas via folhedo e as saídas via produção foi negativo. Por exemplo, em 2003, o balanço de $\mathrm{N}, \mathrm{P}$ e K a $1 \mathrm{~m}$ de distância das fileiras de G. sepium foi $+4,-3,5$ e $-16 \mathrm{~kg} \mathrm{ha}^{-1}$, com valores de -5, $-2,8$ e $-10 \mathrm{~kg} \mathrm{ha}^{-1}$ a $3 \mathrm{~m}$ de distância, respectivamente. Para evitar a diminuição da fertilidade do solo e conferir mais sustentabilidade a esses sistemas, sugere-se que, após o uso da biomassa da G. sepium e da palha do milho como forragem, o esterco produzido pelos animais seja retornado ao solo dos campos de cultivo agrícola.

\section{CONCLUSÕES}

1. A proximidade da G. sepium aumentou significativamente os teores de matéria orgânica leve, $\mathrm{P}$ disponível e K extraível do solo.
2. A matéria orgânica leve do solo foi melhor indicador do que a $\mathrm{CO}$ total para detectar mudanças causadas pelo manejo da cobertura vegetal nas condições do presente estudo.

3. A G. sepium provocou uma diminuição significativa da umidade do solo até distâncias de $1 \mathrm{~m}$ das árvores em relação à posição a $3 \mathrm{~m}$ das árvores.

4. As temperaturas máximas do ar e do solo foram menores embaixo das árvores de G. sepium do que a 1 e $3 \mathrm{~m}$ de distância.

5. A produção de grãos e palha do milho foi maior nas posições mais próximas das fileiras das árvores e diminuiu gradativamente com o aumento da distância das árvores.

\section{AGRADECIMENTOS}

Os autores agradecem ao CNPq, pela concessão da bolsa de estudo, à CAPES, à ONG Assessoria e Serviços a Projetos em Agricultura Alternativa (ASPTA) e ao Inter American Institute for Global Change Research (IAI), pelo apoio financeiro ao presente trabalho; a Francisco Brito e Equipe Técnica da ASPTA, pelo auxílio nas atividades de campo e laboratório, e a Marlon da Silva Garrido, pelas valiosas contribuições realizadas durante a preparação do manuscrito.

\section{LITERATURA CITADA}

ARAUJO FILHO J.A. Manipulação da vegetação lenhosa da caatinga para fins pastoris. Sobral, Embrapa-CNPC. 18p, 1990. (Circular Técnica, 11) 
ASPTA. Conversão agroecológica de sistemas agrícolas familiares no Agreste da Paraíba: uma leitura multidisciplinar a partir do enfoque da produção e do manejo da biomassa. Esperança, ASPTA/CIRAD, 2000. 12p.

BARRIOS, E.; KWESIGA, F.; BURESH, R.J. \& SPRENT, J.I. Light fraction soil organic matter and available nitrogen following trees and maize. Soil Sci. Soc. Am. J., 61:826831, 1997.

BARRETO, A.C. \& FERNANDES, F.M. Cultivo de Gliricidia sepium e Leucaena leucocephala em alamedas visando a melhoria do solos dos tabuleiros costeiros. Pesq. Agropec. Bras., 36:1287-1293, 2001.

BURESH, R.J. \& TIAN, G. Soil improvement by trees in subSaharan Africa. Agrofor. Syst., 38:51-76, 1998.

CORREIA, M.E.F. \& ANDRADE, A.G. Formação de serrapilheira e ciclagem de nutrientes. In: SANTOS, G.A.; FLAVIO A. \& CAMARGO, O., eds. Fundamentos da matéria orgânica do solo: Ecossistemas tropicais e subtropicais. Porto Alegre, Genesis, 1999. 508p.

DANTAS, J.S. Dinâmica da produção e decomposição de folhedo e ciclagem de nutrientes em um ecossistema de caatinga arbórea na Agreste da Paraíba. Areia, Universidade Federal da Paraíba, 2003. 30p. (Monografia do Curso de Agronomia)

EMPRESA BRASILEIRA DE PESQUISA AGROPEQUARIA EMBRAPA. Centro Nacional de Pesquisas de Solos. Sistema Brasileiro de Classificação de Solos. Brasília, 1999a. 412p.

EMPRESA BRASILEIRA DE PESQUISA AGROPEQUARIA EMBRAPA. Informática Agropecuária (Brasília, DF). Manual de análises químicas de solos, plantas e fertilizantes. Brasília, 1999b. 370p.

ESQUIVEL, J.; IBRAHIM, M.; JIMENEZ, F. \& PEZO, D. Distribución de nutrientes en el suelo en sociaciones de poró (Erytrina berteroana), madero negro (Gliricidia sepium) e Arachis pintoi con Brachiaria brizantha. R. Agrofor. la Am., 5:39-43, 1998.

ETCHEVERS, D.J.; FISCHER, R.A.; VIDAL, I.; SAYRE, K.; SANDOVAL, M.A.; OLESCHKO, K. \& ROMAN, S. Labranza de conservación, índices de calidad del suelo y captura de carbono. Colégio de Posgraduados. CIMNYT. Universidad Autónoma de México. In: SIMPOSIUM INTERNACIONAL DE LABRANZA DE CONSERVACIÓN, 2000. Anais. Culiacán-Mazatlán, Sinaloa, 2000. 13p. CDROM

FRAGA, V.S. \& SALCEDO, I.H. Declines of organic nutrient pools in tropical semi-arid soils under subsistence farming. Soil. Sci. Am. J., 68:215-224, 2004.

FRAINDERAICH, N., coord. Atlas solarimétrico do Brasil: Cartas de isolinhas de radiação solar e insolação. Recife, Universidade Federal de Pernambuco, Companhia elétrica do São Francisco (CHESF), CEPEL/ ELECTROBRAS. 2000. v.3. 36p. (Relatório Técnico)

GÓMEZ, M.E. \& PRESTON, T.R. Ciclaje de nutrientes en un banco de proteina de mata Gliricidia sepium. Liv. Res. Rural Develop., 8:6, 1996.
GREGORICH, E.G. \& ELLERT, B.H. Light fraction and macroorganic matter in mineral soils. In: CARTER, M.R., ed. Soil sampling and methods of analysis. Boca Raton, Canadian Society of Soil Science, 1993. p.397-407.

IAEA.Sondas de neutrones y gamma: Sus aplicaciones em agronomia. 2.ed. Viena, 2003. 77p. (Coleccion..., 16).

KWESIGA, F. Perfomance of fifteen povenances of Gliricidia sepium in eastern Zambia. For. Ecol. Manag., 64:161170, 1994

MENDONÇA, E.S.; LEITE, C.L.F. \& NETO, F.P.S. Cultivo do café em sistema agroflorestal: Uma opção para recuperação de solos degradados. R. Árvore, 25:375-383, 2001.

MENEZES, R.S.C. \& SALCEDO, I.H. Influence of tree species on the herbaceous understory and soil chemical characteristics in a silvopastoral system in semi-arid northeastern Brazil. R Bras. Ci. Solo, 23:817-826, 1999.

MENEZES, R.S.C. \& SAMPAIO, E.V.S.B. Agricultura sustentável no semi-árido nordestino. In: OLIVEIRA, T.S.; ROMERO, R.E.; ASSIS Jr., R.N. \& SILVA, J.R.C.S., eds. Agricultura, sustentabilidade e o semi-árido. Fortaleza, Sociedade Brasileira de Ciência do Solo, Universidade Federal do Ceará, 2000. p.20-46.

MENEZES, R.S.C.; SALCEDO, I.H. \& ELLIOTT, E.T. Microclimate and nutrient dynamics in a silvopastoral system of semiarid northeastern Brazil. Agrofor. Syst., $56: 27-38,2002$.

MIELNICZUK, J.; BAYER, C.; VEZZANI, F.M.; LOVATO, T.; FERNANDES, F.F. \& DEBARBA, L. Manejo de solo e culturas e sua relação com os estoques de carbono e nitrogênio. In: CURI, N.; MARQUES, J.J.; GUILHERME, L.R.G.; LIMA, J.M.; LOPES, A.S. \& ALVAREZ V., V.H., eds. Tópicos em ciência do solo. Sociedade Brasileira de Ciência do Solo, 2003. v.3. p.209-248.

MOREIRA, F.M.S. \& SIQUEIRA, J.O. Microbiologia e bioquímica do solo. Lavras, Universidade Federal de Lavras, 2002. 625p.

MURAGE, W.E.; KARANJA, N.K.; SMITHSON, P.C. \& WOOMER, P.L. Diagnostic indicator of soil in productive and non-productive smallholders fields of Kenya's Central Highlands. Amsterdam Agric. Ecosyst. Environ., 79:1-8, 2000.

NAIR, P.K.R. Introduction to agroforestry. Dordrecht, Kluwer Academic Plublishers, 1995. 449p.

SALCEDO, I.H.; TIESSEN, H. \& SAMPAIO, E.V.S.B. Nutrient availability in soil samples from shifting cultivation sites in the semi-arid Caatinga of NE Brazil. Agric. Ecosyst. Environ, 65:177-186, 1997.

SAS. INSTITUTE. SAS Statistical Package, Cary, NC, 1995. Version 6.12.

SCHROTH, G. \& LEHMANN, J. Constrasting effects of roots and mulch form three agroforestry tree species on yield of alley cropepp maize. Agric. Ecosyst. Environ., 54:89$101,1995$.

SIERRA, J.; DULORMME, M. \& DESFONTAINES, L. Soil nitrogen as affected by Gliricidia sepium in a silvopastoral system in Guadeloupe, French Antilles. Agrofor. Syst., 54:87-97, 2002. 
SNYDER, J.D \& TROFYMOW, J.A. A rapid accurate wet oxidation diffusion procedure for determining carbon in plant and soil samples. Comm. Soil Sci. Plant Anal., 15:587-597, 1984

TIESSEN, H.; SALCEDO, I.H. \& SAMPAIO, E.V.S.B. Nutrient and soil organic matter dynamics under shifting cultivation in semi-arid Northeastern Brazil. Agric. Ecosyst. Environ, 38:139-151, 1992.
VITOUSEK, P.M. \& SANFORD, R.L. Nutrient cycling in most tropical Forest. Ann. Rev. Ecol. Sci., 17:137-167, 1986.

WICK, B.; T IESSEN, H. \& MENEZES, R.S.C. Land quality changes following the conversion of natural vegetation into silvo-pastoral systems in semiarid NE Brazil. Plant Soil, 222:59-70, 2000. 